\title{
EDITORIAL
}

\section{El aprendizaje, la enseñanza, los pensamientos y las interacciones en la escuela}

Gran parte de las investigaciones educativas que se publican en revistas de divulgación, institucionales o científicas, suelen estar centradas en el aprendizaje o en la enseñanza, pero también, en alguna medida, en el pensamiento y en las interacciones, o suelen plantear de manera explícita o implícita relaciones con alguno de dichos aspectos. Estos continuos estudios han generado una gran cantidad de posturas, experiencias y evidencias que apuestan por mantener las bases fundamentales de la escuela, la transformación de la educación para dar respuesta a los requerimientos actuales y la consecución de la calidad, la cual puede obedecer al imaginario de los docentes, a las perspectivas institucionales y gubernamentales, a los propósitos de los propios niños y jóvenes o, simplemente, a la búsqueda de innovar, de transformar y de ser creativos.

No obstante, la escuela ha tomado diversas posiciones, ha asumido diversas responsabilidades, ha entrado en la lógica de los resultados y de las perspectivas de quienes desde lo económico orientan y lideran lo educativo a nivel global, y se ha alejado, en diferentes niveles y maneras, de aspectos como los que proponen Masschelein y Simons (2014), quienes señalan que la escuela es una cuestión de suspensión, de profanación, de atención y de mundo, de tecnología, de igualdad, de amor, de preparación y de responsabilidad pedagógica. 


\section{El aprendizaje}

El aprendizaje ha sido uno de los temas claves en el ámbito educativo. Al hablar de procesos de aprendizaje aparecen categorías como la zona de desarrollo próximo (Vigotsky, 1987), a partir de la que se han propuesto posturas vigotskianas, no siempre con profundidad y solidez teórica (Baquero, 1997). De igual manera, hace presencia el aprendizaje significativo, el cual se sustenta en la intención de analizar las maneras y propiedades del aprendizaje para generar cambios cognitivos estables que aporten significado individual y colectivo (Ausubel, 1976; Rodríguez, 2011), al integrar el pensamiento, los sentimientos y las acciones (Novak, 1998).

En la misma dirección, se ha abordado el estudio de las estrategias y estilos de aprendizaje, de manera preponderante desde la psicología, a partir de variables como el contexto, la motivación y la personalidad entre otras (Beltrán, 1993; Schmeck, 1983). En cualquier caso, tal y como establecen Castro y Morales (2015), el aprendizaje debe ser asumido como multifactorial y complejo, motivo por el cual, por ejemplo, se ha dado gran relevancia a los factores ambientales, los cuales tienen el potencial de enseñar por sí mismos.

Lo cierto es que estas perspectivas, al igual que muchas otras que han abordado el aprendizaje, suelen centrarse en el alumno, en el desarrollo de habilidades y competencias, en los resultados e incluso se han asociado al éxito escolar y académico. Si bien es cierto que al pensar en el maestro se mencionan los procesos de formación posgraduada y la formación continuada, no es común que se analice, se estudie y se teorice sobre el aprendizaje de los maestros, a pesar de que muchos autores han abordado el concepto de metaaprendizaje.

Lo interesante de esta reflexión es enfatizar en que el aprendizaje de los maestros es adaptativo, académico, institucional, normativo, político, experiencial, emocional, moral empático, relacional, educativo, pedagógico y creativo, entre muchos otros. Además, el aprendizaje del maestro se construye, se transforma, se deconstruye, se configura y se resignifica en la misma práctica pedagógica, lo que permite una dinámica que posibilita un lugar de indagación 
relevante para los procesos educativos y escolares. Por lo tanto, es fundamental hacer mención a la escuela como forma pedagógica en la que, tal y como plantean Masschelein y Simons (2018), el punto de partida está en la utopía referida a que en la escuela "cualquiera puede aprender cualquier cosa" (p. 21).

\section{La enseñanza}

En cuanto a la enseñanza sucede algo similar. Los estudios se enfocan en los maestros y suelen dejar de lado al alumno, quien también realiza procesos de enseñanza. Sin embargo, esta reflexión se centra de manera particular en uno de los aspectos que inciden de manera significativa en el maestro y, por lo tanto, en la enseñanza. Este aspecto se refiere a las emociones y sentimientos que el docente experimenta antes, durante y después de cada uno de los encuentros con sus alumnos y en la cotidianidad institucional. Desde luego, no se está señalando que el maestro por el hecho de su labor profesional experimente emociones y sentimientos diferentes en lo estructural a cualquier otro profesional, pero sí que su responsabilidad implica tocar la humanidad de los humanos (Royo, 2017) y, en consecuencia, se requiere comprender que en los procesos de pensamiento, interacción, enseñanza y aprendizaje, las emociones intervienen de manera permanente. Por consiguiente, en la educación la gestión emocional es fundamental (Herrera \& Buitrago, 2014).

Una de las emociones que suele hacerse presente con mayor frecuencia en la vida del maestro y en las escuelas es la alegría, ya que muchas situaciones la generan. Entre ellas se puede mencionar la interacción con los demás, el reconocimiento, la sensación de aportar y ayudar, los logros de los discípulos, el sentirse útil y necesario, el estatus social, la confianza que se genera en los demás, entre muchas otras. Pero también suele aparecer el miedo, que - al contrario de la alegría que suele ser pública para poderla compartirse tiende a ocultar y disimular; se intenta ignorarlo o evadirlo.

El miedo se transforma, como casi todo en la vida. Puede llegar a ser adaptativo, si es real; pero también a deshumanizar, si es ilógico (Chacón, 2016). En cualquier caso, como en todas las emociones, los impulsos que se generan pueden llegar a ser positivos o negativos, útiles o inútiles, buenos o malos. Incluso, pueden ser fundamentales 
para la manipulación (Chojin, 2016). Sumado a ello, el miedo se puede aprender desde la infancia, pero también se puede vencer (García, 2016).

El maestro llega a sentir miedo al fracaso, a aquellos estudiantes que lo retan y en ocasiones lo desafían, a los cambios abruptos que no sabe cómo controlar, a ser juzgado, a cometer errores, a no alcanzar el nivel autoimpuesto, a los grupos con los que no logra una adecuada relación, a sentirse menos que otros colegas, al acoso, a la injusticia y a muchas otras circunstancias. Además de estas dos emociones, también circula por las aulas e instituciones el desánimo, al sentir que los cambios que se han venido dando no mejoran las condiciones, que todo en la escuela va empeorando: las actitudes, la sobrecarga laboral, el sentido de vida de muchos estudiantes, los vínculos relacionales con algunos colegas, los mismos resultados, las prácticas negativas de algunos adolescentes, el desinterés por parte de muchos padres y familiares, la apatía de los Estados, líderes y políticos, entre otros.

Esta desesperanza se suele acompañar de impotencia, porque más allá del deseo de que las cosas puedan cambiar, más allá de múltiples esfuerzos por incidir, modificar y transformar, en muchas ocasiones el contexto y los factores que dependen de otros actores, contextos o circunstancias superan al maestro, lo limitany lo reducen, por lo que aparece la desesperanza. En ese momento, la escuela deja de ser grata y la vida del maestro ya está afectada. Por consiguiente, se requiere estar atento al sentido y significado que el maestro da a la enseñanza, a sus emociones y sentimientos, a su profesión y a su vida (Buitrago, Ávila, \& Cárdenas, 2017). Para concluir, es necesario señalar que el maestro experimenta en su cotidianidad múltiples emociones, además con distintos grados de intensidad, pero en esta reflexión, tan solo se quiere hacer mención a algunas emociones que suelen hacerse presentes con cierta frecuencia.

\section{Los pensamientos}

El pensamiento puede ser entendido de diversas maneras si se aborda desde distintas disciplinas y posturas, o desde la perspectiva de Oriente y Occidente. Para Goleman (2003), por 
ejemplo, el pensamiento creativo y espontáneo se propicia cuando las atmósferas de interacción generan sensación de cordialidad y familiaridad. Además, las preguntas interesantes permiten la exploración de diversas respuestas y la apertura para que emerjan los prejuicios que se esconden y limitan el pensamiento.

En Occidente las emociones son públicas, ya que se ponen en evidencia con la expresión facial y corporal, aspecto que es relevante para la comunicación, mientras que los pensamientos son privados. Por el contrario, desde la mirada budista, es poco clara y probable la separación entre las emociones y los pensamientos, ya que estos cuentan con un tono afectivo. En esta misma dirección se ubica la neurociencia moderna, que enfatiza en la relación estrecha entre las emociones y los pensamientos (Ekman, 2003).

Desde esta postura, el Dalai-Lama (2003) plantea algunos interrogantes a la neurociencia: ¿̇el pensamiento está antes de la acción? o ċlos pensamientos anteceden los cambios en el cerebro? Lo cierto es que en la tradición budista, por ejemplo, se trabaja para dirigir de manera voluntaria la actividad cerebral a partir de procesos mentales. Ricard (2003b) complementa con que cada emoción desencadena una secuencia de pensamientos que pueden ser positivos o negativos. Por ello, se debe prestar atención al pensamiento cuando aparece para determinar su configuración y profundidad, con el fin de lograr con la práctica que tanto las emociones como los pensamientos circulen en la mente sin generar distorsión o interrupción.

Por consiguiente, más allá de las perspectivas diversas, opuestas o complementarias respecto al pensamiento, en la línea reflexiva de esta editorial, cabe preguntarse respecto a cómo se está dando el cultivo y desarrollo de pensamiento de los docentes; si en la formación del profesorado se trabaja al respecto o se asume como implícito al abordar textos, autores, conceptos y planteamientos de diversas posturas en el estudio de la pedagogía y de otras disciplinas; de igual manera, si el profesorado se cuestiona y reflexiona sobre sus pensamientos, cómo se configuran, qué incidencia generan, cómo se comparten y cómo se aborda y cómo se propicia el pensamiento en sus alumnos. 


\section{Las interacciones}

La escuela, sin duda, es el lugar privilegiado para las interacciones, para configurar vínculos y para establecer relaciones interpersonales con distintos niveles de intimidad. Para ello, la relación pedagógica se fundamenta, en gran medida, en la comunicación, la cual hace uso de las palabras, los sonidos, los silencios, los gestos, las emociones y el cuerpo (Suárez, Mariño \& Patiño, 2018). Por ello, además de la riqueza e importancia de las palabras y el lenguaje, intervienen otros canales de comunicación, como el oculésico - mirada-, el háptico - tacto y contacto-, el cronémico - los tiempos-, el kinésico - movimientos y gestos-, el proxémico - espacio personal y social-, el diacrítico - simbólico-, el paralingüístico - tono de la voz, ritmo y acentos-y la apariencia.

En este sentido, es importante enfatizar que en la comunicación no existe la no-conducta, debido a que en todo momento se está comunicando algo y, por lo tanto, se está incidiendo en los demás, quienes, por lo general, dan respuesta de manera recíproca (Watzlawick, Beavin\&Jackson,1991).Lociertoesquelacomunicación siempre está matizada, potenciada y en ocasiones manipulada por las emociones. Por ello, una vez más se señala la importancia de que los maestros analicen sus vínculos e interacciones, sus habilidades empáticas, la gestión de sus emociones y la manera en que aportan al desarrollo del ámbito interpersonal de sus alumnos, el cual es fundamental para la estabilidad de los niños, jóvenes y adultos (Buitrago, Herrera \& Cárdenas, 2019; Herrera, Buitrago-Bonilla \& Cepero, 2017). Todo esto se debe a que la convivencia escolar es compleja y dinámica, los conflictos hacen parte de la cotidianidad y las agresiones se revisten de múltiples matices.

\section{Pensamiento final}

La excusa de este texto fue el aprendizaje, la enseñanza, los pensamientos y las interacciones en la escuela, pero la intención de fondo fue la de reflexionar sobre el maestro, quien en ocasiones termina desdibujando su humanidad y su ser tras el imaginario social de su responsabilidad, de sus conocimientos y su desarrollo, de su supuesta superioridad intelectual y experiencial, de su capacidad de sacrificio, y de alguna manera de su estatus social. Ahora bien, no 
pretenden estas palabras difuminar la grandeza de la profesión del maestro, su responsabilidad inherente o el enorme poder de quien orienta a otros; por el contrario, se quiere explicitar la condición humana de maestro y la imprescindible necesidad de ser feliz y disfrutar la escuela.

Para ello, es necesario asumir que ninguna percepción es equitativa o intercambiable y que no siempre se logra expresar con el lenguaje aquello que se desea (Skliar, 2013) o transmitir lo que se quiere con el no lenguaje, en gran medida porque los pensamientos y sentimientos se entretejen, se tensionan y se distensionan para generar nuevas configuraciones, o en ocasiones las mismas, o tal vez nuevas que intentan ser las mismas, o las mismas que pretenden ser diferentes. Es ahí donde la empatía tiene un papel relevante (Herrera, Buitrago \& Ávila, 2016). En consecuencia, el maestro debe consolidar y flexibilizar su pensamiento y llegar, como señalan Espinel y Pulido (2017), a una praxis filosófica que posibilite el cuidado propio, de los demás y de lo otro, al tiempo que se da relevancia a la experiencia y al ensayo. A esta perspectiva se suma lo que plantea Ricard (2003a), respecto a que se requiere construir la felicidad desde el interior y dejar de buscarla afuera, mientras se mantiene una concepción altruista del mundo.

Luego de esta reflexión, presentamos el contenido del número 25 de la Revista Praxis \& Saber. En primera instancia, compartimos con ustedes el artículo Escritura como práctica de sí y escuela rural, de Oscar Pulido Cortés y Paola Lara Buitrago, el cual se orienta a la constitución de sujetos en una escuela rural de Colombia a partir de la escritura como experiencia de sí, la cual se fundamenta en los desarrollos del denominado momento ético de Foucault. De igual manera, el trabajo Lectura, experiencia y aprendizaje: aportes pedagógicos desde "La intuición del instante" de Bachelard, de Carlos Germán Juliao Vargas, analiza la relación de la lectura, la experiencia y el aprendizaje, desde la perspectiva de Bachelard, en la que se aborda la problematización del aprendizaje y la pedagogía del demostrar y del imaginar.

Por su parte, Álvaro Dorado Martínez, Jairo Ascuntar Yandar, Yamile Garcez Muñoz y Lina María Obando Guerrero, en su trabajo Programa de estrategias de aprendizaje para estudiantes de una 
institución educativa, presentan los resultados de una investigación acción realizada en una institución educativa de Sibundoy, Putumayo, la cual implementó un programa de estrategias para el aprendizaje. De igual manera, el trabajo Evaluación del programa de aceleración del aprendizaje: una apreciación estratégica hacia la educación inclusiva en el posconflicto, de Carolina Betancourt Romero, aborda un análisis respecto al cumplimiento de objetivos en una institución educativa de Neiva, a propósito del posconflicto y de la implementación del Programa de Aceleración del Aprendizaje [PAA], implementado en Colombia como modelo flexible.

El manuscrito La riqueza de la evaluación en la escuela, un tesoro no oculto, presentado por Luz Enid Muriel Muñoz, Luz Estela Gómez Vahos y David Alberto Londoño-Vásquez, aborda una reflexión respecto a la evaluación en la escuela, su importancia, su dimensión ética y su relevancia para el aprendizaje, que supera la visión reducida de instrumento de medición y cuantificación. Por su parte, Óscar Leonardo Cárdenas Forero y Marco Tulio Cárdenas Forero, presentan el manuscrito El saber escolar de las artes en la escuela pública colombiana (1800-1850), en el que analizan la presencia y el papel de las artes en la escuela pública colombiana durante el periodo propuesto. Señalan que las disciplinas que se implementaron funcionaron como tecnologías de disciplinamiento y normalización para la estructura establecida en la época.

De otro lado, Jorge Aníbal Rojas Devia y Carlos Arturo Londoño Ramos, en su manuscrito Crisis y praxis filosófica en la educación, reflexionan respecto al aprendizaje filosófico y sus aportes a la enseñanza del filosofar. Parten del hecho de que la enseñanza de la filosofía y el filosofar son básicos para la formación de las personas y para la adquisición de competencias desde la praxis filosófica. Luego, Gerson Aurelio Maturana Moreno y María Lisbet Lombo Sánchez, en su trabajo Inteligencia naturalista: efectos sobre el pensamiento crítico y las necesidades de cognición, analizan los resultados de la implementación de un programa pedagógico fundamentado en la inteligencia naturalista, el cual se enfocó en el desarrollo de habilidades de pensamiento crítico para fortalecer el pensamiento en estudiantes de cuarto de primaria de una institución educativa pública de Bogotá. 
El artículo Currículo narrativo y tecnologías en la formación docente: reflexiones teórico-conceptuales, de Maria Elizabeth Bianconcini de Almeida y Alessandra Rodrigues, aborda el concepto de currículo narrativo de Ivor Goodson y las tecnologías digitales de información y comunicación [TDIC], e integra aspectos como aprendizaje narrativo y capital narrativo.

Lucy González Lerma y Carlos Lugo Silva, en su artículo Fortalecimiento de la práctica docente con Learning Analytics: estudio de caso, realizan un análisis sobre el uso de un software para la gestión de aula CloudLabs en una institución educativa de Bogotá, de manera particular en el área de ciencias naturales en los grados de sexto a décimo. Posterior a ello, el manuscrito Innovación curricular en la formación en turismo: un enfoque teórico-conceptual, de Marysol Castillo Palacio, Luz Marina Ardila Barragán y Andrés José Castrillón Muñoz, plantea una reflexión acerca de artículos publicados durante el periodo de 2008-2018 en Scopus y Science Direct, respecto a la innovación del currículo en la formación en turismo, en particular en cuanto a competencias, habilidades y características.

Para concluir este número, Walter Omar Kohan presenta la traducción de Paulo Freire: otras infancias para la infancia, en donde se plantean algunas aportaciones de Paulo Freire sobre la infancia. Allí se aborda una perspectiva relevante en la que se supera la concepción de etapa cronológica, para dar paso a una perspectiva referente a la fuerza de la vida. Estimados lectores, esperamos que este nuevo número de la revista sea de su agrado y siga aportando hallazgos y resultados derivados de la investigación educativa y siga enriqueciendo el debate respecto a la educación, la pedagogía y la didáctica.

\section{Dr. Rafael Enrique Buitrago Bonilla}

Editor Invitado

Universidad Pedagógica y Tecnológica de Colombia

Grupos de Investigación Cacaenta y DEDICA Línea de Investigación Emociones \& Educación https://orcid.org/oooo-0oo1-7553-6473 https://doi.org/10.19053/22160159.v11.n25.2020.10580 


\section{Referencias}

Ausubel, D. (1976). Psicología educativa. Un punto de vista cognoscitivo. México: Trillas.

Baquero, R. (1997). Vigotsky y el aprendizaje escolar. Argentina: Aique Grupo Editor S.A.

Beltrán, J. (1993). Procesos, estrategias y técnicas de aprendizaje. Madrid: Síntesis.

Buitrago, R.,Ávila,A., \& Cárdenas, R. (2017). El sentidoyel significado atribuido a las emociones por el profesorado en formación de la Universidad Pedagógica y Tecnológica de Colombia. Contextos Educativos, 20, 77-93. http://doi.org/10.18172/con.2998

Buitrago, R., Herrera, L., \& Cárdenas, R. (2019). Coeficiente emocional en niños y adolescentes de Boyacá, Colombia. Estudio comparativo. Praxis \& Saber, 10(24), 45-68. https:// doi.org/10.19053/22160159.v10. n25.2019.10002

Castro, M., \& Morales, M. (2015). Los ambientes de aula que promueven el aprendizaje, desde la perspectiva de los niños y niñas escolares. Revista Electrónica Educare, 19(3), 1-32. http://dx.doi.org/10.15359/ree.19-3.11

Chacón, F. (2016). Presentación II: Vencer el miedo. En G. Fouce (Coord.), Psicología del miedo (pp. 11-12). Colombia: Alfaomega \& Grupo 5 .

Chojin, (2016). La energía y el miedo. En G. Fouce (Coord.), Psicología del miedo (pp. 19-21). Colombia: Alfaomega \& Grupo 5 .

Dalai-Lama. (2003). ¿Qué son las emociones destructivas? La anatomía de las aflicciones mentales. En D. Goleman (Ed.), Emociones Destructivas, cómo entenderlas y superarlas (pp. 55-70). Barcelona: Editorial Kairós.

Ekman, P. (2003). La universalidad de las emociones. En D. Goleman (Ed.), Emociones Destructivas, cómo entenderlas y superarlas (pp. 71-93). Barcelona: Editorial Kairós.

Espinel, O., \& Pulido, O. (2017). Enseñanza de la filosofía: Entre 
experiencia filosófica y ensayo. Universitas Philosophica, 69(34), 121-142. https://doi.org/10.11144/Javeriana.uph34-69. efee

García, L. (2016). Fundamentos biológicos y psicológicos del miedo. En G. Fouce (Coord.). Psicología del miedo (pp. 23-30). Colombia: Alfaomega \& Grupo 5.

Goleman, D. (Ed.). (2003). Emociones Destructivas, cómo entenderlas y superarlas. Barcelona: Editorial Kairós.

Herrera, L., \& Buitrago, R. (2014). Emociones, inteligencia emocional, educación y profesorado. En L. Herrera (Coord.), Retos y desafios actuales de la Educación Superior desde la perspectiva del profesorado universitario (pp. 179-203). Madrid: Síntesis.

Herrera, L., Buitrago, R., \& Ávila, A. (2016). Empathy in future teachers of the Pedagogical and Technological University of Colombia. New Approaches in Educational Research, 5(1), 3037. https://doi.org/10.7821/naer.2016.1.136

Herrera, L., Buitrago-Bonilla, R., \& Cepero S. (2017). Emotional Intelligence in Colombian Primary School Children. Location and Gender. Universitas Psychologica, 16(3), 1-10. https://doi. org/10.11144/Javeriana.upsy16-3.eips

Masschelein, J., \& Simons, M. (2014). Defensa de la Escuela, una cuestión pública. Argentina: Miño y Dávila Editores.

Masschelein, J., \& Simons, M. (2018). La lengua en la escuela: ¿alienante o emancipadora?. En J. Larrosa (Ed.), Elogio de la Escuela (pp. 19-39). Argentina: Miño y Dávila Editores.

Novak, J. (1998). Learning, Creating and Using Knowledge. New Jersey: Lawrence Erlbaum Associates.

Ricard, M. (2003a). Plaidoyer pour le bonheur. París: NiL Éditions.

Ricard, M. (2003b). ¿Qué son las emociones destructivas? Una psicología budista. En D. Goleman (Ed.), Emociones destructivas, cómo entenderlas y superarlas (pp. 47-55). Barcelona: Editorial Kairós.

Rodríguez, M. (2011). La teoría del aprendizaje significativo: una 
revisión aplicable a la escuela actual. Revista Electrònica d'Investigació i Innovació Educativa i Socioeducativa, 3(1), 29-50.

Royo, M. (2017). Nuevas perspectivas en la formación inicial del profesorado. En P. Darder (Coord.), La formación emocional del profesorado (pp. 169-190). Barcelona: Editorial Octaedro.

Schmeck, R. (1983). Learning styles of college students. En R. Dillon, \& R. Schmeck (Eds.), Individual differences in cognition: Vol 1. Nueva York: Academic Press.

Skliar, C. (2013). No tienen prisa las palabras. Argentina: Miño y Dávila Editores.

Suárez, M., Mariño L., \& Patiño, O. (2018). Voces, gritos y reclamos: de la exclusión a la emancipación. Una forma de concluir lo inconcluso. En M. Suárez, \& Ó. Pulido (Coords.), Infancia, diversidad y filosofía. Voces gritos y reclamos (pp. 81-100). Tunja: Editorial UPTC.

Vigotsky, L. (1987). Historia del desarrollo de las funciones psicológicas superiores. La Habana: Ed. Científico-Técnica.

Watzlawick, P., Beavin, J., \& Jackson, D. (1991). Teoría de la comunicación humana. Barcelona: Herder. 